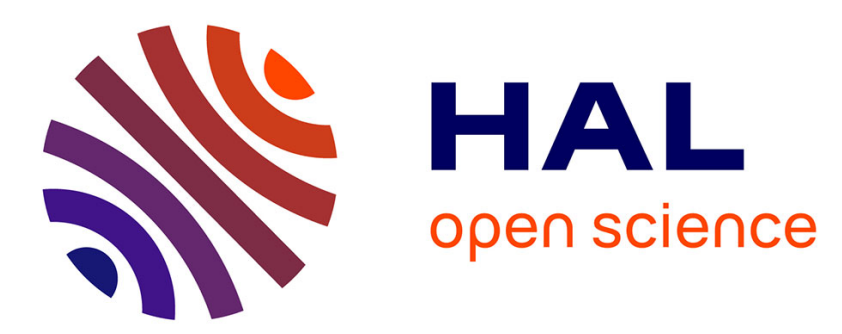

\title{
The effect of the cutoff frequency on the sound production of a clarinet-like instrument
}

E Petersen, P. Guillemain, J. Kergomard, T Colinot

\section{To cite this version:}

E Petersen, P. Guillemain, J. Kergomard, T Colinot. The effect of the cutoff frequency on the sound production of a clarinet-like instrument. Journal of the Acoustical Society of America, 2019, 10.1121/1.5111855 . hal-02188757

\section{HAL Id: hal-02188757 https://hal.science/hal-02188757}

Submitted on 26 Jul 2019

HAL is a multi-disciplinary open access archive for the deposit and dissemination of scientific research documents, whether they are published or not. The documents may come from teaching and research institutions in France or abroad, or from public or private research centers.
L'archive ouverte pluridisciplinaire HAL, est destinée au dépôt et à la diffusion de documents scientifiques de niveau recherche, publiés ou non, émanant des établissements d'enseignement et de recherche français ou étrangers, des laboratoires publics ou privés. 
The effect of the cutoff frequency on the sound production of a clarinet-like instrument

E. Petersen, ${ }^{1}$ P. Guillemain, ${ }^{1}$ J. Kergomard,${ }^{1}$ and T. Colinot $^{1}$

Aix Marseille Univ, CNRS, Centrale Marseille, LMA, UMR 7031, Marseille, France $^{\text {a) }}$ 
The input impedance of woodwind instruments is characterized by at least two bands due to the lattice of open toneholes, a stop band at low frequencies and a pass band at higher frequencies where the acoustic energy is able to propagate past the first open tonehole and into the lattice. The cutoff frequency that separates these two bands is an approximate value that is determined by the geometry of the lattice of open toneholes. It is expected that the frequency at which the stop band transitions to the pass band affects the sound produced by the instrument, but it is not known how this frequency affects the competition between self-sustained oscillation and radiation. A simplified model of a clarinet-like resonator is conceived such that the first input impedance peak and the cutoff frequency can be independently chosen. Experimental prototypes are built and their measured impedance are compared with the simulations. Resonators with very similar low frequency behavior, but very different cutoff frequencies, are then compared using digital synthesis to evaluate the influence of the cutoff frequency on sound production. The cutoff frequency impacts the synthesized pressure and acoustic volume velocity in the mouthpiece, particularly regarding the spectral content at high frequencies.

a) petersen@lma.cnrs-mrs.fr; 


\section{INTRODUCTION}

Reed instruments are typically characterized by two main components: the reed and flow rate as a nonlinear excitation mechanism and the main bore of the instrument as a passive acoustic resonator that responds to and influences the excitation mechanism. Under normal playing conditions the resonator responds to an injection of acoustical energy from the exciter by radiating sound, but also facilitates the self-sustained oscillation of the exciter. ${ }^{1}$ The input impedance, determined by the specific geometry of the bore and tonehole network, is a standard way to characterize the acoustic behavior of the resonator. A resonator with a series of open toneholes exhibits a well-known behavior called the tonehole lattice cutoff frequency, below which a lattice wave sampled at discrete points is evanescent, while above, a lattice wave can propagate further into the bore. ${ }^{2,3}$ Below the cutoff frequency the resonator has an effective length that is approximately the length between the input and the first open tonehole. In the case of a cylindrical bore, due to constructive interference of the reflected waves, the input impedance is characterized by well-defined, nearly harmonically spaced maxima expected of a quarter-wave resonator. Above the cutoff frequency, the effective length of the resonator is a complicated function of frequency, so there is less organized constructive and destructive interference, and the impedance is characterized by attenuated peaks that are not harmonically related to the first impedance peak.

It is often assumed that the cutoff frequency behavior is related to the perceptual characteristics of the sound produced by an instrument. ${ }^{1,4}$ However, it is not known precisely in what way the cutoff frequency affects the production and radiation of sound. Therefore, 
it is worth investigating how the frequency at which cutoff occurs, and the severity of the cutoff behavior on the input impedance, could impact the self-sustained oscillation and the temporal and spectral characteristics of the produced sound. Similarly, the cutoff frequency may impact characteristics of sound radiation such as directivity and total radiated power with respect to frequency. The complicated behavior associated with sound production and radiation are linked to one another through the competition between the acoustical energy that is retained within the resonator, which facilitates production, and that which is radiated into the surrounding environment.

The goal of this article is to evaluate how the cutoff frequency impacts different aspects of sound production and timbre features. To reduce the number of parameters that influence the acoustical behavior of a real instrument, the current work (except Sect. III C) considers resonators composed of a cylindrical main bore terminated by a lattice of geometrically regularly spaced toneholes. This simplified version of a clarinet resonator allows direct analysis of the effects of the cutoff frequency without added complications such as irregular tonehole lattices, conical segments, and undercut toneholes, as is the case for actual woodwind instruments.

Section II contains a review of the Transfer Matrix Method (TMM $)^{5-7}$ for simulating the input impedance of a pipe, including the related Transfer Matrix Method with external Interactions (TMMI) ${ }^{8}$ which accounts for mutual radiation impedance when there are more than a single radiating aperture (toneholes and open termination of the main bore). Section III details the design and experimental verification of a cylinder and tonehole lattice resonator for which it is possible to specify both the frequency of the first impedance peak 
and the cutoff frequency. The effects on sound production, for three different resonators, are compared using digital synthesis in Section IV. The main focus of this section is to characterize the internal sound field, as well as the transfer function between internal and radiated time averaged intensity. Examples of synthesized pressure and velocity waveforms are furnished as supplementary materials online. Conclusions and proposals for future work are covered in Section V.

\section{INPUT IMPEDANCE CALCULATIONS VIA THE TRANSFER MATRIX} METHOD WITH EXTERNAL INTERACTIONS

While the TMM is elegant, simple, and efficient, it ignores the effects of external interactions due to toneholes radiating simultaneously into the same space. A variant of the TMM is the Transfer Matrix Method with external Interactions (TMMI). ${ }^{8,9}$ This method accounts for propagation within the main bore while also accounting for the mutual impedance between apertures ( $N$ toneholes and the bore end) that are assumed to radiate into the same space, and therefore have a mutual influence.

The TMMI allows the calculation of the acoustic pressure vector $P$ and the acoustic flow vector $U$ through each aperture of the resonator. They are related to the source term by

$$
\begin{gathered}
U=[\mathbb{I}+\mathbb{Y}(\mathbb{Z}+\mathbb{B})]^{-1} U^{\mathrm{s}} \\
P=\mathbb{Y}^{-1}\left(U^{\mathrm{s}}-U\right),
\end{gathered}
$$

where $U^{\mathrm{s}}$ is the source vector, of dimension $N+1$ and with only one non zero element: $U_{n}^{\mathrm{s}}=\delta_{n, 1} . \mathbb{I}$ is the identity matrix, and $\mathbb{Y}$ is a tri-diagonal admittance matrix, derived from the $2 \times 2$ transfer matrices of the main bore between toneholes. A cylinder is added to 
account for the section of pipe separating the mouthpiece from the first open tonehole of a real instrument. $\mathbb{B}$ is the impedance matrix for the acoustic mass of each tonehole: the definition includes many equations, and can be found in the appendix of Lefebvre et al. ${ }^{8}$ $\mathbb{Z}$ is the radiation impedance matrix. Along the diagonal, it accounts for the classical self radiation impedance of each aperture, and for the mutual radiation impedance between two apertures, it is assumed that they radiate as a monopole in an half infinite space. ${ }^{8,10,11}$ The input impedance is calculated from the knowledge of the quantities $P_{1}$ and $U_{1}$.

At low frequencies the monopoles can be assumed to radiate into a full space, however, the uncertainty of the mutual loading approximation is greater than the factor of two between half space and full space radiation.

The TMMI impedance calculation yields subtly different results when compared with that of the standard TMM, as seen in Fig. 4. The two methods are in good agreement at low frequencies. At higher frequencies, the TMMI reduces the height of the resonances and reduces the depth of the anti-resonances, which can be interpreted as a reduction in the standing wave ratio of the resonator and an increase of the radiation losses in the system. Knowing $P$ and $U$ at the location of the toneholes allows for the calculation of external values such as directivity and the pressure waveform at a given external location, topics that are outside the scope of this paper but useful for future work. 


\section{RESONATOR DESIGN}

\section{A. Periodic lattice of holes and resulting cutoff frequency}

The basic resonator considered in the current work is designed to have a desired first impedance resonance frequency at $f_{1}$ and desired cutoff frequency at $f_{\mathrm{c}}$ that can be manipulated independently from one another by varying the geometry. This is achieved by concatenating a cylinder of length $L$, which largely determines $f_{1}$, to a lattice of geometrically regular toneholes that imposes the cutoff behavior at $f_{\mathrm{c}}$, as shown in Fig. 1(b). This is similar to the resonator design in the dissertation by Worman. ${ }^{12}$ While not explained in great detail, it is important to note that simple geometric calculations must include low frequency length corrections, defined in Section III B, due to the lattice of toneholes as well as the more familiar radiation length correction at each open tonehole.

The analytic formulations used in this method allow only for the frequencies $f_{1}$ and $f_{\mathrm{c}}$ to be independently determined (see Eq. (2) and (4)). The second impedance peak of two resonators with different cutoff frequencies occur at nearly the same frequency because there is very low inharmonicity due to the cylindrical portion of the resonator, and the use of identical toneholes. Dimensions and acoustic features of sample resonators are provided in Appendix A.

\section{B. Geometrically regular lattice}

A geometrically (and therefore also acoustically) regular lattice is constructed by repeating the basic cell shown in Fig. 1 (b) to create the lattice shown in Fig. 1 (a). If the cells are 


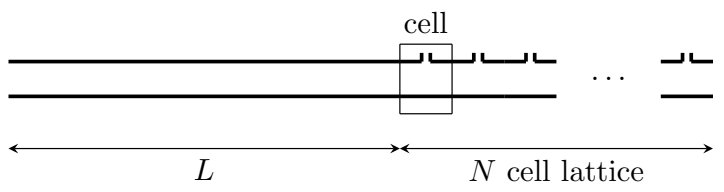

(a)

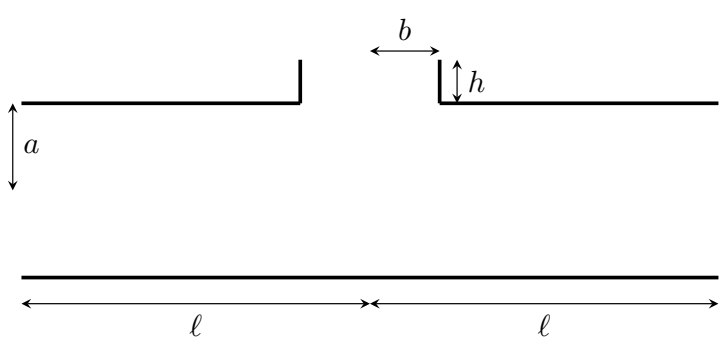

(b)

FIG. 1. Pipe and lattice resonator (a) constructed from identical cells (b).

all identical and their number infinite, the global cutoff frequency, which can be estimated from impedance measurements or simulations, will be equal to the local cutoff frequency. The local cutoff frequency is approximated by the Helmholtz resonance of a single cell whose main bore is closed at the extremities, ${ }^{13}$

$$
\omega_{\mathrm{c}}=2 \pi f_{\mathrm{c}} \approx \frac{c}{\ell} \frac{1}{\sqrt{\left(\frac{c}{\ell}\right)^{2} m_{h} C_{a}+1 / 3}},
$$

where $C_{a}=2 \ell \pi a^{2} / \rho c^{2}$ is the acoustic compliance of the main pipe, $m_{h}=\rho h / \pi b^{2}$ is the acoustic mass of the tonehole, and $a, \ell, b$ and $h$ are the geometric dimensions of the cell shown in Fig. 1 (b).

The exact local cutoff frequency for a cell in an infinite lattice can be calculated from

$$
{ }_{c}^{\ell} \cot (k \ell)=\omega m_{h} C_{a}
$$

which is derived by solving the pressure and flow transfer matrix equations assuming either Dirichlet or Neumann boundary conditions within the main bore. ${ }^{9}$ This exact form differs by less than $1 \%$ from the approximate form given in Eq. (2) for an equivalent Helmholtz resonator of dimensions used in the current article. To impose a desired local cutoff frequency it is sufficient to find a combination of dimensions $a, \ell, b$, and $h$, accounting for the length 
corrections due to the inertial effect of adjacent fluid and the radiation impedance of the tonehole, that result in the desired resonance. Therefore, it is possible to design a lattice to have a desired global cutoff frequency by manipulating the dimensions of the constituent cells to have the same local cutoff frequency. To impose the frequency of the first impedance peak $f_{1}$, a cylinder of length $L$ is concatenated to the lattice and treated like a quarter-wave resonator,

$$
f_{1}=\frac{c}{4 L_{a}}
$$

where $L_{a}=L+\Delta \ell$ is an effective acoustic length that accounts for the presence of a lattice of open toneholes. The lattice modifies the radiation impedance of the cylinder as a mass-like term, and is treated as a length correction

$$
\Delta \ell=\ell \sqrt{1+\frac{\ell^{2}}{c^{2}} C_{a} m_{h}}
$$

which must be included in addition to the physical length of the main cylinder when determining the appropriate length that results in the desired frequency $f_{1} \cdot{ }^{1}$ A strong cutoff frequency behavior is observed with as few as three open toneholes, however, the frequency at which cutoff occurs tends to be somewhat higher than that calculated in Eq. (2) when the lattice is comprised of a small number of holes, see Fig. 2. Lattices with 10 toneholes exhibit a very strong cutoff behavior at the desired frequency. Three resonators, all with first resonance frequencies $f_{1}=170 \mathrm{~Hz}$ and with cutoff frequencies $f_{c}=1000,1500$, and $2000 \mathrm{~Hz}$, are used frequently in the current paper and will therefore be referred to as resonators $\mathcal{R}_{1.0}, \mathcal{R}_{1.5}$ and $\mathcal{R}_{2.0}$. All three have ten toneholes in addition to the open termination of the pipe. The input impedance of all three were simulated and two $\left(\mathcal{R}_{1.0}, \mathcal{R}_{2.0}\right)$ were 


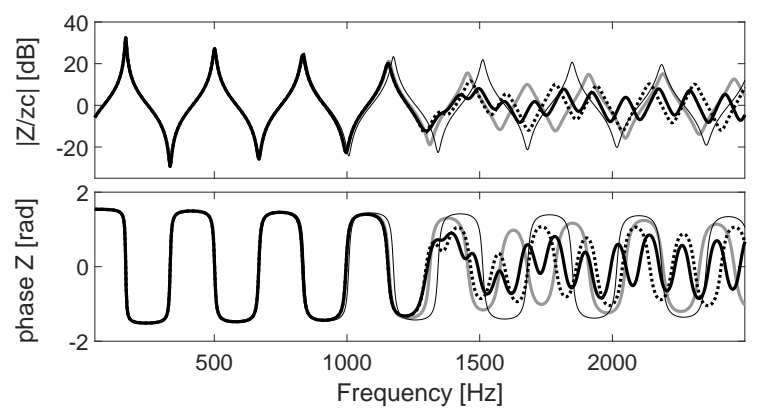

FIG. 2. Simulated impedance curves of resonators with $f_{1}=170 \mathrm{~Hz}, f_{c}=1350 \mathrm{~Hz}$, and varying number of toneholes (thin solid black: cylinder; thick solid grey: 4 holes; thick dashed black: 10 holes; thick solid black: 20 holes). The cutoff frequency $f_{c}=1350 \mathrm{~Hz}$ was chosen to occur at an impedance minimum so that the effect on the next maximum can be more easily distinguished. Lattices with 10 holes exhibit very strong cutoff behavior.

constructed physically (dimensions provided in Appendix A) and compared with simulation in Section IIID.

\section{Acoustic regularity}

Although the analysis based on digital synthesis in Section IV A is limited to a tonehole network composed of geometrically regular cells, Moers and Kergomard ${ }^{13}$ show that it is possible to define an acoustic regularity for which each constituent cell has the same Helmholtz resonance, but not necessarily the same geometry. In that article, they consider the inverse problem of determining the division of an acoustically regular lattice that results in the expected global cutoff frequency. In the present paper, the problem is solved directly by designing each cell to be geometrically different, except the bore radius, but have a common Helmholtz resonance, and therefore have an acoustic regularity that results in the 
desired global cutoff frequency. The individual cells have identical main bore internal radii $a$, and the tonehole radii and heights $b, h$ taken from the clarinet dimensions provided in Appendix A1 of Moers and Kergomard. ${ }^{13}$ Once a desired cutoff frequency is set, the length of pipe $\ell$ on each side of the symmetric cells is deduced by Eq. (2). This acoustically regular lattice can then be constructed with a leading pipe to create the desired first resonance peak described in Section III B. Figure 3 shows that a global cutoff frequency is attained using an acoustically (but not geometrically) regular lattice. As with a geometrically regular lattice, the low frequency behavior of the acoustically regular lattice is largely unchanged between resonators with different cutoff frequencies. Specifically, the height and frequency of the first two impedance peaks are negligibly different for different resonators. This similarity between geometrically and acoustically regular resonators suggests that the choice to use geometrically regular resonators in this work is a reasonable simplification of real instruments, which exhibit substantial acoustic regularity. Next, the effect of the order of acoustically regular cells is examined by calculating the input impedance for randomized permutations of the cell locations. Figure 3 demonstrates that the order of the cells in the lattice does not greatly influence the frequency at which cutoff occurs and, as above, has a negligible influence on the height and frequency of the first two impedance peaks. This is a result of work by Fürstenberg ${ }^{14}$ demonstrating exceptions to the nonexistence of pass bands in an infinite one-dimensional random medium. ${ }^{13,15}$ It is worth noting that, above the cutoff frequency and up to approximately $2000 \mathrm{~Hz}$, the impedance of each resonator, regardless of the cell order, follows similar and slowly diverging paths. This is unexpected and suggests that, for frequencies just above cutoff, the input impedance behavior is determined by global 


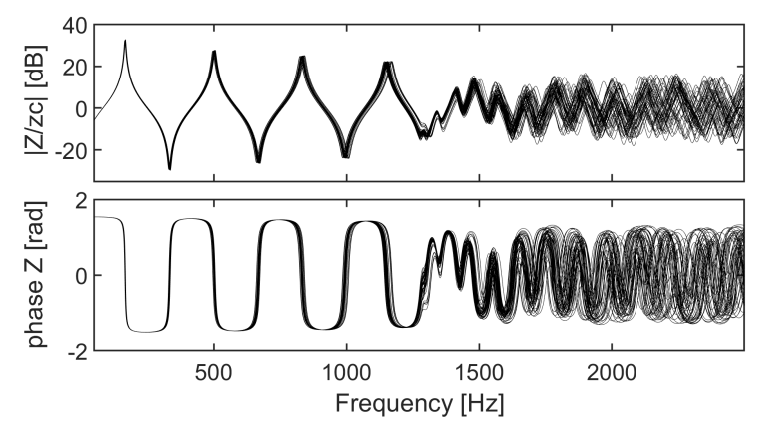

FIG. 3. Multiple simulated input impedances for resonators with acoustically regular lattices and random cell orders (50 permutations). The target frequencies are $f_{1}=170 \mathrm{~Hz}$ and $f_{\mathrm{c}}=1350 \mathrm{~Hz}$.

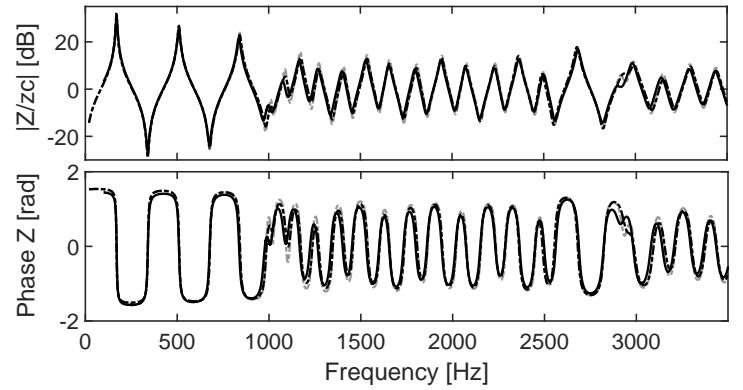

(a)

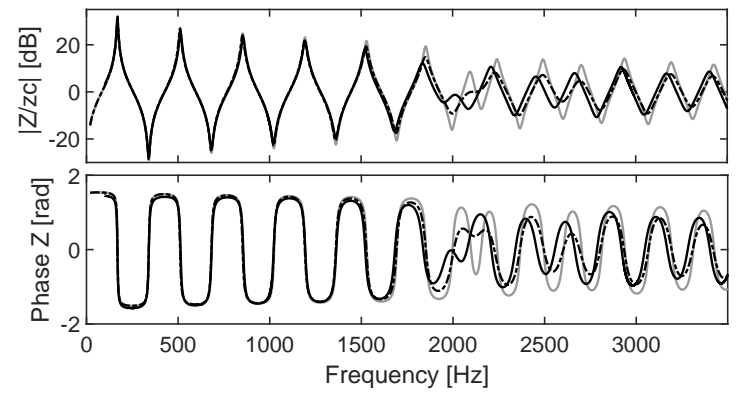

(b)

FIG. 4. Input impedance of $\mathcal{R}_{1.0}$ (a) and $\mathcal{R}_{2.0}$ (b). Grey: TMM simulation; dashed black: TMMI simulation; solid black: experimental.

\section{Experimental results of a simple resonator}

The simplified pipe-lattice resonator described in the previous section was tested experimentally by measuring the input impedance of two polyamide 6 tubes where the lengths $L$ 
and tonehole network geometries are designed to have first impedance resonances at $170 \mathrm{~Hz}$ and cutoff frequencies at $1000 \mathrm{~Hz}$ and $2000 \mathrm{~Hz}\left(\mathcal{R}_{1.0}, \mathcal{R}_{2.0}\right)$, according to Eq. (2) and (4). The dimensions are summarized in Appendix A. The input impedance was measured using an impedance measurement device developed at CTTM. ${ }^{16}$ Figure 4 shows that the target first impedance resonance and cutoff frequencies are attained by both simulation and measurements. This demonstrates the relevance of the model and accuracy of the measurement methodology. The better agreement between simulation and measurement for the $f_{c}=1000$ $\mathrm{Hz}$ resonator is a consequence of the greater distance between holes, and not a general trend related to the cutoff frequency of a resonator. A second stop band in the impedance of $\mathcal{R}_{1.0}$ is visible around $2500 \mathrm{~Hz}$ both in the simulation and measurement. The discrepancy between simulation and measurements at high frequencies may be due to slight imprecision of the physical geometries of the experimental pipes, inaccuracies of the measurement hardware, or higher order phenomena not included in the simulation. These differences do not affect the conclusions, which are based on the location of the cutoff frequency and not the precise high frequency behavior.

\section{EFFECTS OF CUTOFF FREQUENCY USING DIGITAL SYNTHESIS}

Digital sound synthesis is a convenient method to evaluate the playing characteristics of a real or hypothetical resonator, and to compare two or more different resonators, because it can synthesize time domain acoustic pressure and volume velocity waveforms inside the mouthpiece for a wide range of control parameters. One can then compute descriptors of the waveform that are used to quantify playing characteristics, with respect to the control 
parameters, such as playing frequency, spectral centroid, and attack time. These playing descriptors are functions of the dimensionless control parameters excitation pressure $\gamma$, and embouchure $\zeta$ (defined below), but also are dependent on the reed parameters and input impedance of the resonator. ${ }^{17,18}$ It is assumed that $\gamma$ and $\zeta$ control timbre features of a given instrument, and are therefore useful parameters to evaluate the connection between sound production and sound descriptors. It is important to note that synthesized waveforms do not necessarily match those of a real instrument because the values of physical parameters, particularly regarding the reed dynamics, are not precisely known. However, digital synthesis is a reasonable tool to compare the behavior of different resonators. The following sections are based on synthesis for resonators $\mathcal{R}_{1.0}, \mathcal{R}_{1.5}$ and $\mathcal{R}_{2.0}$ as defined in Section III B.

\section{A. Digital synthesis model}

The digital synthesis model used in this work combines the discretization scheme of Guillemain, ${ }^{17}$ with the use of the reflection function to model the response of the resonator. ${ }^{19,20}$ Its purpose is to solve, in the domain of time sampled signals, a system of three equations and three unknowns giving the values at sample number $n$ as functions of the past known values of the three variables. For that purpose, we start with the continuous time model describing the resonator, the reed dynamics, and the nonlinear relationship between pressure and flow in the mouthpiece. 


\section{Continuous time model}

The continuous time reed instrument model used in this work is comprised of three main equations (Eq. (7), (9), and (10)). The three variables are the dimensionless pressure, flow rate, and reed displacement, respectively

$$
\left\{\begin{array}{l}
\tilde{p}(t)=p(t) / p_{M} \\
\tilde{u}(t)=Z_{c} u(t) / p_{M}, \\
\tilde{x}(t)=x(t) / H,
\end{array}\right.
$$

where $Z_{c}$ is the characteristic impedance, $p_{M}$ is the pressure difference needed to close the reed completely in a static situation, and $H$ is the distance between the tip of the reed and the mouthpiece lay at equilibrium. The value $\tilde{x}=-1$ corresponds to the closure of the reed. The first equation corresponds to the movement of the reed which consists of a damped spring-mass driven by the pressure differential between the mouth of the musician and the mouthpiece

$$
\left\{\begin{array}{l}
\frac{1}{\omega_{r}^{2}} \frac{d^{2} \tilde{x}(t)}{d t^{2}}+\frac{q_{r}}{\omega_{r}} \frac{d \tilde{x}(t)}{d t}+\tilde{x}(t)=\tilde{p}(t)-\gamma \text { if } \tilde{x}>-1, \\
\frac{d \tilde{x}(t)}{d t}=0 \text { if } \tilde{x}<-1
\end{array}\right.
$$

where $\omega_{r}$ is the resonance angular frequency of the reed, $q_{r}$ is the damping coefficient, and the parameter $\gamma=p_{m} / p_{M}$ is the dimensionless pressure in the mouth of the musician. Following values similar to other articles, ${ }^{18}$ the synthesis results presented in this paper assume $\omega_{r}=2 \pi f_{r}=2 \pi 1500(\mathrm{rad} / \mathrm{s})$ and $q_{r}=0.4$ (dimensionless). The second line of Eq. (7) corresponds to the limitation of the reed displacement by the mouthpiece lay. There exist more refined models of the reed that include dynamical behavior and the collision of the reed 
and the lay. ${ }^{22-24}$ These models, however, are unnecessary for the current work which aims to efficiently compare the response of different resonators, not predict absolute behavior. The nonlinear relationship relating the input flow $u(t)$ as a function of the pressure differential and the reed position is

$$
\tilde{u}(t)=u_{b}(t)+u_{r}(t)
$$

where

$$
\left\{\begin{array}{l}
u_{b}(t)=\zeta(1+x(t)) \operatorname{sign}(\gamma-\tilde{p}(t)) \sqrt{|\gamma-\tilde{p}(t)|} \\
u_{r}(t)=\lambda_{c} \frac{d x(t)}{d t}
\end{array}\right.
$$

The variable $\zeta=Z_{c} w H \sqrt{2 / \rho p_{M}}$ is the dimensionless embouchure parameter, depending on the width $w$ and height $H$ of the reed channel cross section when the reed is at rest, and is proportional to the square root of $H$ because $p_{M}$ is a function of $H$. The total flow rate is the sum of the flow computed from the stationary Bernoulli equation $u_{b}$ and the flow due to the movement of the reed $u_{r}$, depending on the speed of the reed and a parameter $\lambda_{c}$ that characterizes the effective vibrating surface of the reed. ${ }^{25}$

The third principle equation represents the passive role of the resonator and is expressed by its input impedance. Following McIntyre ${ }^{26}$ and Gazengel, ${ }^{19}$ a straightforward change of variables in the Fourier domain, using wave variables rather than Kirchhoff variables, leads to the expression of the backward pressure wave at time $t$ in the temporal domain

$$
p^{-}(t)=\left(r * p^{+}\right)(t)
$$


where $p^{-}(t)=(\tilde{p}(t)-\tilde{u}(t)) / 2$ and $p^{+}(t)=(\tilde{p}(t)+\tilde{u}(t)) / 2$ are the backward and forward pressure waves in the resonator. The operator $*$ represents continuous time convolution and $r$ is the reflection function of the resonator, obtained from the input impedance by

$$
r=\mathcal{F}^{-1}\left(\frac{Z(\omega)-Z_{c}}{Z(\omega)+Z_{c}}\right)
$$

where $\mathcal{F}^{-1}$ is the inverse Fourier transform. The advantage of using the reflection function rather than the impulse response is that it is assumed to decrease much faster in time, and can therefore be truncated to save computation time.

\section{Discrete model}

The continuous time model of the complete instrument is discretized so that temporal synthesis can be simulated by a computer. Therefore, a strictly causal formulation must be found so that every variable at a given time sample can be computed from the previous values of all variables. Equation (7) is discretized using the finite difference scheme ${ }^{20}$ such that

$$
x[n]=b_{1 r} p[n-1]+a_{1 r} x[n-1]+a_{2 r} x[n-2],
$$

where the tilde notation is omitted for dimensionless variables in the discrete formulation ( $p[n]$ is the $n^{\text {th }}$ sample of the dimensionless pressure signal). The coefficients $b_{1 r}, a_{1 r}$ and $a_{2 r}$ are given in Guillemain et al. ${ }^{20}$ In order to ensure the limitation of the reed displacement, the following condition is added 


$$
\text { if } x[n]<-1 \text {, then } x[n]=x[n-1] \text {. }
$$

Once the position of the reed at sample $n$ is known, the reed flow is computed using the finite difference scheme

$$
u_{r}[n]=\lambda(x[n]-x[n-1])
$$

The parameter $\lambda=\lambda_{c} f_{s}$ is fixed at the value -0.7 as in Coyle et al. ${ }^{18}$ based on the measurements available in the literature. ${ }^{25-28}$ The third equation involving the reflection function can be reformulated using the discrete convolution product

$$
\left(r * p^{+}\right)[n]=\sum_{i=0}^{\infty} r[i] p^{+}[n-i]
$$

where $r$ is the discrete version of the reflection function, which is causal. For the numerical application, the reflection function is deduced from the computed input impedance, which is windowed down to the characterstic impendance by $8 \mathrm{kHz}$, well below the first nonplanar mode and following recommendations from Gazengel. ${ }^{19}$ Above this frequency the impedance is completed by the characteristic impedance until the Nyquist frequency, before computing the reflection function by inverse Fourier transform. Only the first $D=3000$ elements of the reflection function are kept for the synthesis, chosen to be long enough so that it does not disturb the low frequency content of the resonator's response. Specifically, the impulse response is a sum of exponentially damped sinusoids and this choice of $D$ ensures that the time response of the lower mode, with the highest quality factor, is not truncated. The 
backward pressure wave corresponds is written as

$$
p^{-}[n]=\sum_{i=0}^{D} r[i] p^{+}[n-i]
$$

Separating this equation into a strictly causal and an instantaneous part and substituting for $p$ and $u, p^{-}[n]$ becomes

$$
\frac{1}{2}(p[n]-u[n])=\frac{1}{2} r[0](p[n]+u[n])+\frac{1}{2} \sum_{i=1}^{D} r[i](p[n-i]+u[n-i])
$$

where $r[0]$ is the first sample of the discrete reflection function. This yields $p[n]$ as a function of $u[n]$ and the past of $p$ and $u$

$$
p[n](1-r[0])=u[n](1+r[0])+\sum_{i=1}^{D} r[i](p[n-i]+u[n-i]),
$$

The final pressure expression is as follows:

$$
p[n]=\frac{1+r[0]}{1-r[0]} u[n]+\frac{1}{1-r[0]} \sum_{i=1}^{D} r[i](p[n-i]+u[n-i]) .
$$

Using Eq. (19), it is possible to implement the temporal synthesis scheme described in Guillemain et al., ${ }^{20}$ because it is in the form $p[n]=b_{c_{o}} u[n]+V$ where $\mathrm{V}$ is a known quantity at sample $n$.

\section{B. Results}

Synthesized time and frequency domain waveforms of the internal pressure and flow for resonators $\mathcal{R}_{1.0}, \mathcal{R}_{1.5}$, and $\mathcal{R}_{2.0}$ are shown in Fig. 5 , and available to listen to online ${ }^{21}$. The waveforms are taken from the periodic and steady state region of the synthesis, which is calculated with control parameters $\gamma=0.45, \zeta=0.45$. In this representation the signals 

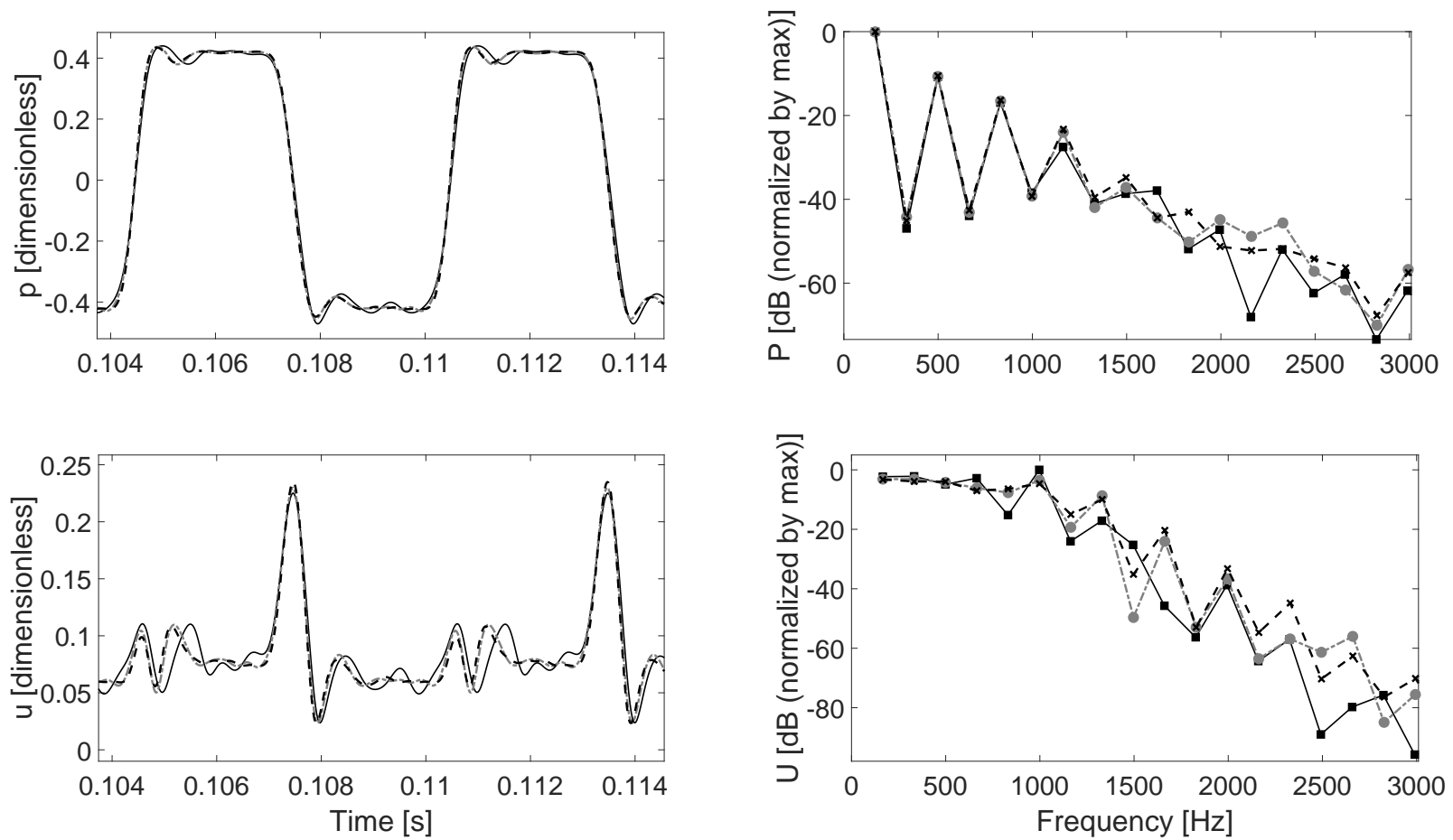

FIG. 5. Synthesized (dimensionless) pressure (top) and flow (bottom) inside mouthpiece for resonators $\mathcal{R}_{1.0}$ (solid black), $\mathcal{R}_{1.5}$ (dash-dot gray), and $\mathcal{R}_{2.0}$ (dashed black) in the time (left) and frequency (right) domains. Control parameters are $\gamma=\zeta=0.45$, and reed resonance $f_{r}=1500 \mathrm{~Hz}$.

from the three resonators have been arbitrarily shifted in time by less than one cycle, so that they overlay one another, facilitating visual comparison. The timing difference of the pressure and flow signals is small, and may result from a small change in playing frequency. There is a subtle difference between both the pressure and volume velocity for resonators $\mathcal{R}_{1.0}, \mathcal{R}_{1.5}$, and $\mathcal{R}_{2.0}$. The biggest visual difference is in the volume velocity waveform, in which resonator $\mathcal{R}_{1.0}$ has larger amplitude and lower frequency rippling when compared with resonators $\mathcal{R}_{1.5}$ and $\mathcal{R}_{2.0}$. A simple interpretation, examined in Section IV B 1, is that there is more energy in the high frequency harmonics in the waveforms computed from resonators with higher cutoff frequencies. The height of the peaks that are the main feature 


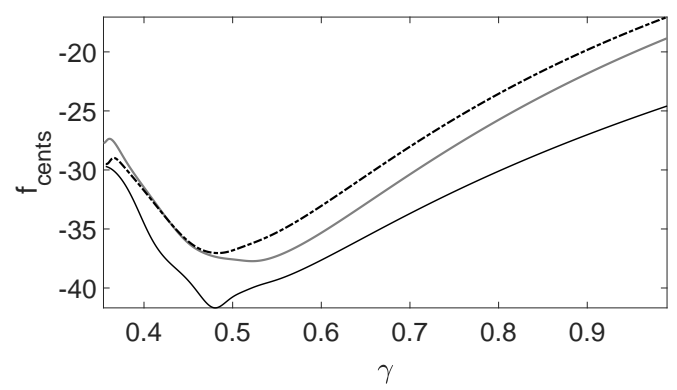

FIG. 6. Synthesized playing frequency in cents, normalized by the first impedance peak frequency, for resonators $\mathcal{R}_{1.0}, \mathcal{R}_{1.5}$, and $\mathcal{R}_{2.0}$ with solid black, solid grey, dashed black lines, respectively.

in the velocity waveform, corresponding to the closing of the reed, also changes between the different resonators.

\section{Frequency domain characteristics}

Frequency domain characteristics are computed directly from the steady state portion of the synthesized waveforms $p$ and $u$ inside the mouthpiece, discarding the attack transient. The playing frequency, $f_{\mathrm{p}}$, of resonators $\mathcal{R}_{1.0}, \mathcal{R}_{1.5}$, and $\mathcal{R}_{2.0}$, as a function of $\gamma$ with constant $\zeta=0.45$, are displayed in Fig. 6 . The values are presented in cents, relative to the frequency of the first impedance peak, such that

$$
f_{\text {cents }}=1200 \log _{2}\left(\frac{f_{\mathrm{p}}}{f_{1}}\right) .
$$

It is seen that resonators with higher cutoff frequencies also have playing frequencies that are higher by a few cents. In the beating reed regime, approximately $\gamma>0.5$, the difference between resonators $\mathcal{R}_{1.0}$ and $\mathcal{R}_{2.0}$ is greater than five cents, therefore possible large enough to be perceived by a human. ${ }^{29}$ This is not due only to the difference in inharmonicity between the first and second peaks, verified through analytical formulations, ${ }^{18}$ in which 


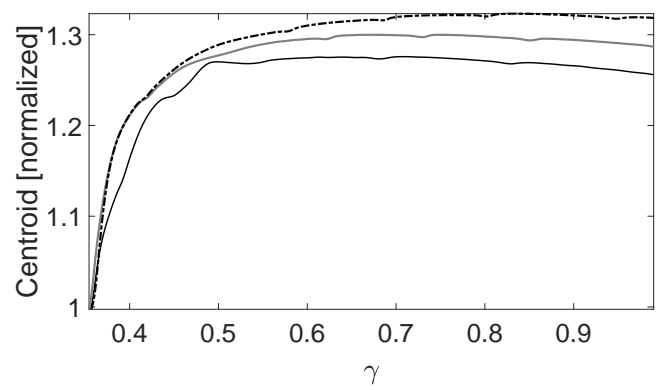

FIG. 7. Synthesized spectral centroid, normalized by the playing frequency, for resonators $\mathcal{R}_{1.0}, \mathcal{R}_{1.5}$, and $\mathcal{R}_{2.0}$ with solid black, solid grey, dashed black lines, respectively.

the inharmonicity of these resonators results in a maximum of 2.5 cents difference between resonators $\mathcal{R}_{1.0}$ and $\mathcal{R}_{2.0}$. However, the tonehole lattice also changes the inharmonicity of higher resonances of the input impedance below the cutoff frequency. This could influence the playing frequency, particularly at high values of $\gamma$ and $\zeta$.

To define a single value descriptor, the spectral centroid ${ }^{30}$ is calculated as

$$
\text { centroid }=\frac{\sum_{m=1}^{M} f(m) G(m)}{\sum_{m=1}^{M} G(m)},
$$

where $G(m)$ is the single sided power spectrum corresponding to the Fast Fourier Transform frequency bins $f(m)$ up to $f(M)=8 \mathrm{kHz}$, at which point the assumptions made in the impedance simulation regarding thermo-viscous losses and radiation are no longer valid. This definition results in a single number for a given spectrum with units of $\mathrm{Hz}$, which follows the 'center of mass' interpretation of the spectral content. The spectral centroid values, normalized by the playing frequency at each combination of control parameter and for each resonator, are shown in Fig. 7. The spectral centroid is higher for resonators with higher cutoff frequencies, even after adjusting for the playing frequency. 

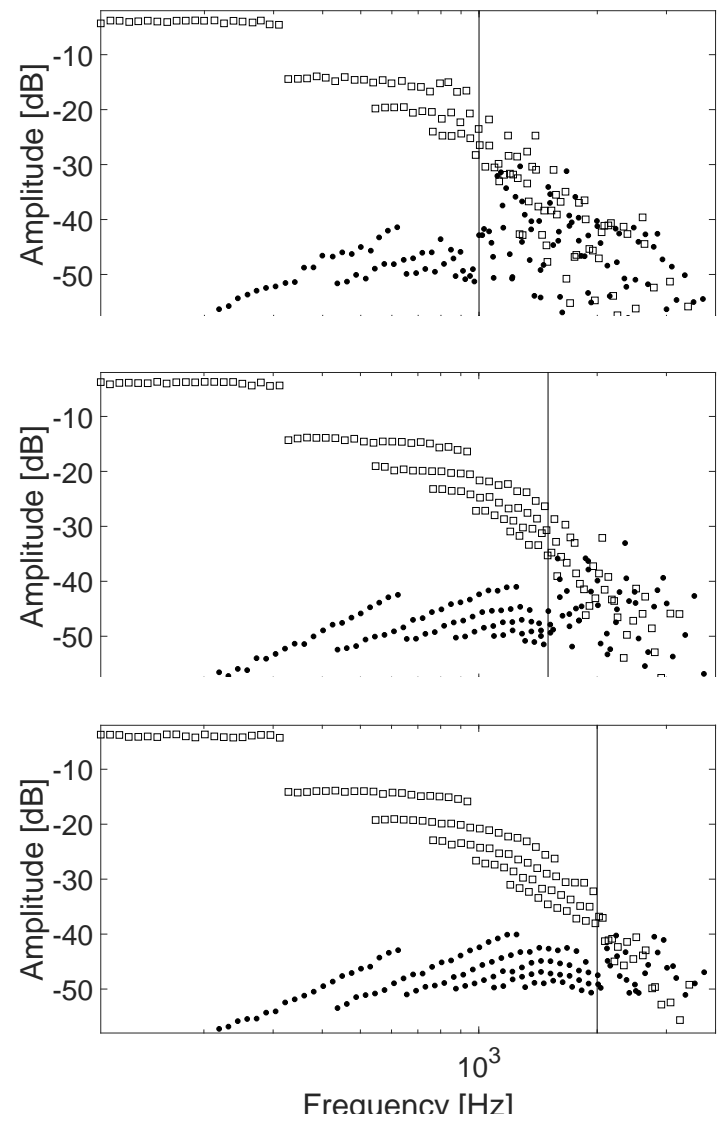

FIG. 8. Synthesized $(\zeta=0.45, \gamma=0.55)$ spectra of the mouthpiece pressure for resonators $\mathcal{R}_{1.0}, \mathcal{R}_{1.5}$, and $\mathcal{R}_{2.0}$ for 20 fundamental frequencies ranging chromatically from $110 \mathrm{~Hz}$ to $330 \mathrm{~Hz}$. Odd and even harmonics denoted by squares and circles, respectively.

To evaluate the influence on the distribution of harmonics in synthesized signals, the input impedance was simulated for a wide range of resonators with fundamental frequencies of 20 consecutive semitones ranging from 110 to $330 \mathrm{~Hz}$ for all three cutoff frequencies 1000, 1500, and $2000 \mathrm{~Hz}$. This results in 60 simulated resonators: 20 of each type $\mathcal{R}_{1.0}, \mathcal{R}_{1.5}$ and $\mathcal{R}_{2.0}$. The first impedance peak frequency is manipulated by changing the length of the cylinder between the mouthpiece and the first open tonehole, leaving the lattice of 10 open toneholes unchanged. Digital sound synthesis was then calculated for the 60 resonators, 
using a beating regime value $\zeta=0.45, \gamma=0.55$ in order to generate more even harmonics. A peak detection scheme was applied to the spectra of the internal pressure where the square and circular data markers correspond to the odd and even harmonics. The results are plotted in Fig. 8 for resonators of type $\mathcal{R}_{1.0}, \mathcal{R}_{1.5}$ and $\mathcal{R}_{2.0}$ in top, middle, and bottom, respectively, and vertical lines denote the cutoff frequency of the resonators used in each figure.

For all resonators shown here, regardless of their cutoff frequency, the odd harmonics that are below the cutoff frequency have approximately the same amplitude climbing the semitone scale. Around and above the cutoff frequency the amplitudes of the odd harmonics decreases, and the strong organization disappears as also seen in Fig. 5, which implies that the complicated input impedance above cutoff frequency has a randomizing effect on the production of odd harmonics. This can be interpreted as the system no longer behaving as a quarter-wave resonator at these frequencies. Additionally, the even harmonics generally grow in amplitude as a function of frequency, and display a marked increase in amplitude for frequencies higher than the cutoff frequency. This implies that below the cutoff frequency, the spectral content of the waveforms produced by resonators with different cutoff frequencies is relatively similar, but that the high frequency spectra, and hence the perceived sound, can be quite different above the cutoff frequency. Specifically, the plots show more energy in even harmonics above the cutoff frequency.

The same 60 resonators are compared in terms of the time averaged intensity at input of the resonators and the the time averaged intensity summed over each radiating aperture, 
shown in Fig. 9. The time averaged intensity is defined as

$$
\begin{aligned}
<I_{\text {in }}> & =\frac{1}{2} \operatorname{Re}\left[P_{0} U_{0}^{*}\right], \\
<I_{\text {rad }}> & =\sum_{n=1}^{M} \frac{1}{2} \operatorname{Re}\left[P_{n} U_{n}^{*}\right],
\end{aligned}
$$

where $M=N+1$ is the number of toneholes $N$ plus the open end of the resonator. This provides a direct comparison between the energy that remains inside the resonator and contributes to the auto-oscillation and the total energy that exits the resonator. A comparison of the left and right panels shows that the time averaged intensity of the fundamental frequency at the input is on the order of $40 \mathrm{~dB}$ higher than the output, suggesting that the resonators are inefficient sound sources, regardless of cutoff frequency. Note that the level of the fundamental frequency input intensity is not horizontal as it is for pressure in the mouthpiece. The output intensity of the fundamental frequency increases proportional to $\omega^{2}$. This is consistent with known monopole radiation efficiency and bolsters the claim that, below the cutoff frequency, the main radiating source can be treated as a monopole at the location of the first open tonehole. Above the cutoff frequency the time averaged intensity is approximately the same for the input and output. This implies that the resonators are more efficient sources above the cutoff frequency.

It is concluded that the cutoff frequency affects the spectral content of waveforms within the mouthpiece by both demarcating the frequency at which even harmonics develop, as well as shifting the spectral centroid higher for resonators with higher cutoff frequencies. 

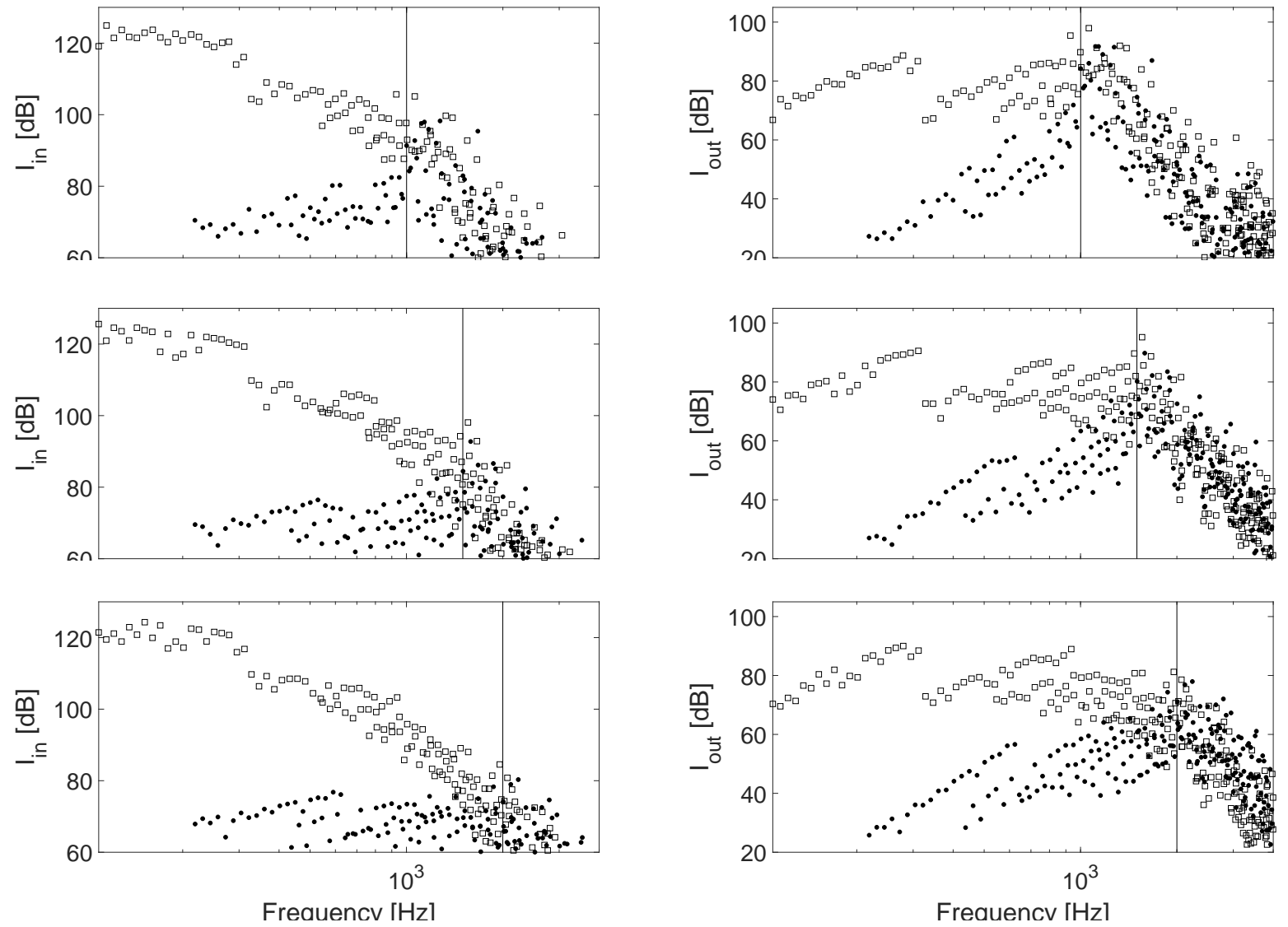

FIG. 9. Time averaged intensity passing through the entrance of the resonator (left) and the summed over ten toneholes and exit of resonator (right). Resonators and synthesis control parameters are the same as in Fig 8. The cutoff frequency for each set of resonators is marked by a vertical line.

\section{Attack time}

To evaluate the influence of the cutoff frequency on the transient portion of the waveform, the time evolution of the waveform as the musician increases the blowing pressure from atmospheric pressure $(\gamma=0)$ to the steady state value of $\gamma$ is simulated. To avoid discontinuities in the onset pressure, the transition from $\gamma=0$ to the steady state value 


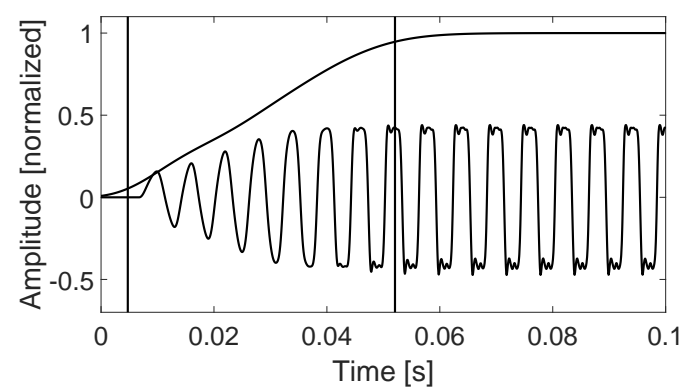

FIG. 10. Synthesized pressure waveform and sum of time evolving harmonics for resonator $\mathcal{R}_{1.0}$ with $5 \%$ and $95 \%$ thesholds marked.

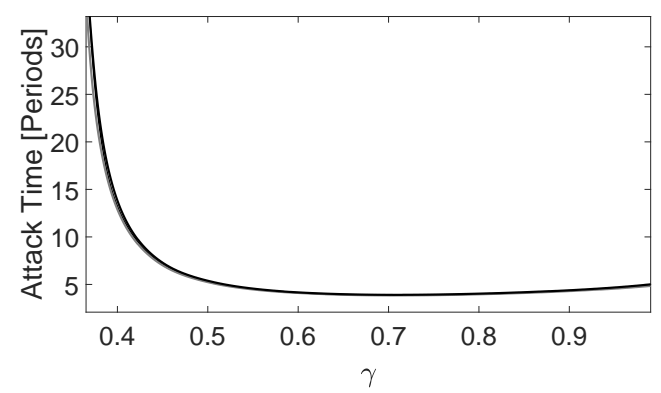

FIG. 11. Synthesized attack time normalized by period of playing frequency for resonators $\mathcal{R}_{1.0}, \mathcal{R}_{1.5}$ and $\mathcal{R}_{2.0}$ with solid black, solid grey, dashed black lines, respectively. Variation in attack times due to cutoff frequency is generally less than $10 \%$.

follows the curve of the first half of a Hann window. In the current work the duration of the transition is 201 samples, corresponding to about five milliseconds for a sampling rate of 44100 samples per second. To define the attack time, the time domain pressure signal is decomposed into its constituent harmonics, each of which evolves as a function of time. To include frequencies above the cutoff frequency of all resonators, 16 harmonics are considered. The harmonics are calculated by an inverse Fourier transform on windowed versions of the spectrum around each harmonic. This estimates the instantaneous amplitude and frequency 
of each harmonic for the duration of the signal, including during the attack transient. The sum of the squared amplitude of these bands is proportional to the total energy in the signal at a given instant, and the energy in the steady state portion of the signal can be determined by choosing this value well into the steady state portion of the signal. The beginning of the attack is determined when the signal first passes a threshold that is a specific, arbitrary fraction of the final value, and the end of the signal attack is defined as the last time that the sum is outside a threshold from the steady state value. Onset threshold values of 5 and 10 percent, and ending threshold values of 90 and 95 percent have been investigated. Figure 10 shows a typical pressure waveform onset (in this case for $\mathcal{R}_{1.0}$ ), with the sum of the first 16 harmonics, normalized to have a maximum value of unity, superimposed. The threshold at $5 \%$ and $95 \%$ are marked in bold lines. It is found in this work that the attack time is linearly related to the choice of threshold, and therefore any of these four choices (5-90, 5-95, 10-90, 10-95) is reasonable for comparing the attack time of different resonators. Figure 11 shows the attack time normalized by the period of the playing frequency as a function of $\gamma$ with constant $\zeta=0.45$. Thresholds of $5 \%$ and $95 \%$ are used to define the onset and saturation of the waveform. The attack time of resonators $\mathcal{R}_{1.0}, \mathcal{R}_{1.5}$, and $\mathcal{R}_{2.0}$ are sufficiently similar as to be difficult to discern. The difference between attack times of different resonators is less than $10 \%$ of a period for $\gamma>0.5$, and up to 2.5 periods for $\gamma<0.5$. However, for low blowing pressures the attack time is of the order of tens of periods, so the difference of 2.5 periods is still a small percentage of the total attack time. It is concluded that the cutoff frequency does not strongly impact the attack time of a given resonator which, in a musical context, may be related to the ease of playing. 


\section{CONCLUSION}

The acoustic resonators developed in this work are adapted for studying the effect of the cutoff frequency on sound production and, potentially, radiation. The simple nature of these resonators, for which it is possible to independently vary the frequencies of both the first impedance peak and the cutoff frequency, is particularly useful because the low frequency behavior (below cutoff) is largely the same for resonators with very different high frequency behavior. The data resulting from either digital sound synthesis or experimental measurements can then be used to directly assess the effect of the cutoff frequency on the sound production and resulting external sound field.

It is found that the cutoff frequency does affect the synthesized waveform within the mouthpiece, particularly the spectral characteristics. Resonators with a higher cutoff frequency have higher playing frequencies, and higher spectral centroids, when compared with resonators with lower cutoff frequencies. Furthermore, even harmonics tend to be excited above the cutoff frequency of a given resonator. However, the differences between internal waveforms in resonators with different cutoff frequencies is subtle. Furthermore, the cutoff frequency of a resonator is not found to have a significant influence on the attack time, showing that one measure of the "playability" of a clarinet-like instrument relies more on the low frequency response of the resonator. This implies that an instrument maker may be able to make modifications to the geometry of an instrument that results in a different cutoff frequency, without the risk of greatly changing the sound production qualities of the instrument. 
In contrast to the relatively small effect of the cutoff frequency on sound production, the cutoff frequency has a larger impact on the high frequency spectral characteristics and radiation. The radiation efficiency, calculated as the ratio of the time averaged intensity at the input of the instrument with that summed over the radiating apertures, is higher above the cutoff frequency than below. Above the cutoff frequency the resonators produce more even harmonics and radiate more efficiently, implying that the cutoff frequency could have a substantial impact on the perceived timbre of different instruments, or different notes on the same instrument.

This, along with the small impact on sound production, indicates that the cutoff frequency may be used to change the timbre characteristics of an instrument without altering its sound production qualities.

Future work includes extending the synthesis analysis to the external sound field to evaluate the effect of the cutoff frequency on radiated sound, for which Fig. 9 provides an initial answer. This includes directivity of the source due to multiple radiating apertures. A characterization of the external sound field can then be compared with the internal sound field to evaluate the competition between sound production and radiation of clarinet-like musical instruments.

\section{ACKNOWLEDGMENTS}

This work has been partly supported by the french Agence Nationale de la Recherche (ANR16-LCV2-0007-01 Liamfi project). The authors would also like to thank Patrick Sanchez and Guy Rabau for their help with the impedance measurements. 
TABLE I. Dimensions for two prototype resonators with the first impedance peak $f_{1}$ at $170 \mathrm{~Hz}$ and cutoff frequencies $f_{\mathrm{c}}$ at $1000 \mathrm{~Hz}$ and $2000 \mathrm{~Hz}$. Simulated and measured input impedance are provided in Fig. 4.

\begin{tabular}{c|ccccc}
\hline \hline Resonator & $L[\mathrm{~mm}]$ & $a[\mathrm{~mm}]$ & $\ell[\mathrm{mm}]$ & $b[\mathrm{~mm}]$ & $h[\mathrm{~mm}]$ \\
\hline $\mathcal{R}_{1.0}$ & 436.7 & 7.0 & 32.8 & 4.0 & 8.4 \\
$\mathcal{R}_{2.0}$ & 470.1 & 7.0 & 8.9 & 4.0 & 8.4 \\
\hline \hline
\end{tabular}

\section{APPENDIX A: MEASUREMENTS: PROCEDURE AND MATERIALS}

Two resonators were realized to experimentally verify the feasibility of designing resonators with independently chosen first input impedance peaks and cutoff frequencies. The resonators were made by drilling holes in polyamide 6 tubes, the dimensions of which are summarized in Table I. The input impedance was then measured using the impedance measurement device developed at CTTM in hemi-anechoic conditions. ${ }^{16}$ Table II summarizes the first two impedance peak frequencies of resonators $\mathcal{R}_{1.0}, \mathcal{R}_{1.5}$, and $\mathcal{R}_{2.0}$ used for the digital synthesis. The inharmonicity, in cents, is defined as

$$
\text { inharmonicity }=1200 \log _{2}\left(\frac{f_{2}}{3 f_{1}}\right) .
$$


TABLE II. Frequencies of the first two impedance peaks and inharmonicity of resonators $\mathcal{R}_{1.0}$, $\mathcal{R}_{1.5}$, and $\mathcal{R}_{2.0}$

\begin{tabular}{|c|c|c|c|c|}
\hline Resonator & $f_{c}[\mathrm{~Hz}]$ & $f_{1}[\mathrm{~Hz}]$ & $f_{2}[\mathrm{~Hz}]$ & inharm [cents] \\
\hline $\mathcal{R}_{1.0}$ & 1000 & 170 & 511.6 & 5.4 \\
\hline $\mathcal{R}_{1.5}$ & 1500 & 170 & 513.0 & 10.2 \\
\hline $\mathcal{R}_{2.0}$ & 2000 & 170 & 513.1 & 10.5 \\
\hline
\end{tabular}

\section{References}

${ }^{1}$ A. H. Benade, Fundamentals of Musical Acoustics (Oxford University Press, London, 1976).

${ }^{2}$ L. Brillouin and M. Parodi, Wave propagation in periodic structures (Mc Graw Hill, New York, NY, USA, 1946).

${ }^{3}$ A. H. Benade, "On the mathematical theory of woodwind finger holes," J. Acoust Soc. Am. 32, 1591-1608 (1960).

${ }^{4}$ J. Wolfe and J. Smith, "Cutoff frequencies and cross fingerings in baroque, classical, and modern flutes," J. Acoust Soc. Am. 114(4), 2263-2272 (2003).

${ }^{5}$ G. R. Plitnik and W. J. Strong, "Numerical method for calculating input impedances of the oboe," J. Acoust Soc. Am. 65(3), 816-825 (1979).

${ }^{6}$ R. Causse, J. Kergomard, and X. Lurton, "Input impedance of brass musical instrumentscomparison between experiment and numerical models," J. Acoust Soc. Am. 75(1), 241- 
254 (1984).

${ }^{7}$ D. Keefe, "Woodwind air column models," J. Acoust Soc. Am. 88, 35-51 (1990).

${ }^{8}$ A. Lefebvre, G. P. Scavone, and J. Kergomard, "External tonehole interactions in woodwind instruments," Acta Acustica united with Acustica 99, 975-985 (2013).

${ }^{9}$ A. Chaigne and J. Kergomard, Acoustics of Musical Instruments (Springer-Verlag, New York, 2016), (English translation).

${ }^{10}$ R. L. Pritchard, "Mutual acoustic impedance between radiators in an infinite rigid plane," J. Acoust Soc. Am. 32(6), 730-737 (1960).

${ }^{11} \mathrm{P}$. Rucz, "A finite element approach for the calculation of self and mutual radiation impedances of resonators," J. Acoust Soc. Am. 143(4), 2449-2459 (2018).

${ }^{12}$ W. E. Worman, "Self-sustained nonlinear oscillations of medium amplitude in clarinet like systems," Ph.D. thesis, Case Western Reserve University, 1971.

${ }^{13}$ E. Moers and J. Kergomard, "On the cutoff frequency of clarinet-like instruments. geometrical versus acoustical regularity," Acta Acustica united with Acustica 97, 984-96 (2011).

${ }^{14}$ H. Fürstenberg, "Noncommuting random matrix products," Trans. Am. Math. Soc. 108, 377-428 (1963).

${ }^{15} \mathrm{P} . \mathrm{W}$. Anderson, "Absense of diffusion in certain random lattices," Phys. Rev. 109, 14921505 (1958).

${ }^{16}$ J. C. L. Roux, M. Parchebat, and J. Dalmont, "A new impedance sensor for industrial applications," Conf. Proceedings: Acoustics 2012 (2012). 
${ }^{17} \mathrm{P}$. Guillemain, "A digital synthesis model of double-reed wind instruments," EURASIP Journal on Applied Signal Processing 7, 990-1000 (2004).

${ }^{18}$ W. L. Coyle, P. Guillemain, J. Kergomard, and J.-P. Dalmont, "Predicting playing frequencies for clarinets: A comparison between numerical simulations and simplified analytical formulas," J. Acoust Soc. Am. 138(5), 2770-2781 (2015).

${ }^{19}$ B. Gazengel, J. Gilbert, and N. Amir., "Time domain simulation of single-reed wind instrument. From the measured input impedance to the synthesis signal. Where are the traps?," Acta Acustica united with Acustica 3, 445-472 (1995).

${ }^{20} \mathrm{P}$. Guillemain, J. Kergomard, and T. Voinier, "Real-time synthesis of clarinet-like instruments using digital impedance models," J. Acoust Soc. Am. 118, 483-494 (2005).

${ }^{21}$ E. Petersen, "[url will be inserted by aip]," Synthesized mouthpiece waveforms as audio for pressure SuppPubmm1-3, acoustic flow SuppPubmm4-6, and time derivative of pressure plus flow SuppPubmm7-9 (an approximation of raidated sound), for resonators with cutoff frequencies of 1000,1500 , and $2000 \mathrm{~Hz}$.

${ }^{22}$ F. Avanzini and M. Walstijn, "Modelling the mechanical resonse of the reed-mouthpiecelip system of a clarinet. part i. a one-dimensional distributed model," Acta Acustica united with Acustica 90, 537-547 (2004).

${ }^{23}$ M. Walstijn and F. Avanzini, "Modelling the mechanical resonse of the reed-mouthpiecelip system of a clarinet. part ii: A lumped model approximation," Acta Acustica united with Acustica 93, 435-446 (2007). 
${ }^{24}$ S. Bilbao, A. Torin, and V. Chatziioannou, "Numerical modeling of collisions in musical instruments," Acta Acustica united with Acustica 101, 155-173 (2015).

${ }^{25}$ J.-P. Dalmont, P. Guillemain, and P.-A. Taillard, "Influence of the reed flow on the intonation of the clarinet," in Acoustics 2012 (2012).

${ }^{26}$ M. E. McIntyre, R. T. Schumacher, and J. Woodhouse, "On the oscillations of musical instruments," J. Acoust Soc. Am. 74, 1325-1345 (1983).

${ }^{27}$ J. P. Dalmont, B. Gazengal, J. Gilbert, and J. Kergomard, "Some aspects of tuning and clean intonation in reed instruments," Applied Acoustics 46, 19-60 (1995).

${ }^{28}$ J. Dalmont, J. Joël, and S. Ollivier, "Nonlinear characteristics of single-reed instruments: Quasistatic volume flow and reed opening measurements," J. Acoust Soc. Am. 114(4), $2253-2262(2003)$.

${ }^{29}$ E. G. Shower and R. Biddulph, "Differential pitch sensitivity of the ear," J. Acoust Soc. Am. 3, 275-287 (1931).

${ }^{30} \mathrm{~S}$. McAdams, J. W. Beauchamp, and S. Meneguzzi, "Discrimination of musical instrument sounds resynthesized with simplified spectrotemporal parameters," J. Acoust Soc. Am. 105(2), 882-897 (1999).

\section{List of Figures}

1 Pipe and lattice resonator (a) constructed from identical cells (b)........ 8 


\section{Effect of cutoff frequency on woodwinds}

2 Simulated impedance curves of resonators with $f_{1}=170 \mathrm{~Hz}, f_{c}=1350 \mathrm{~Hz}$, and varying number of toneholes (thin solid black: cylinder; thick solid grey: 4 holes; thick dashed black: 10 holes; thick solid black: 20 holes). The cutoff frequency $f_{c}=1350 \mathrm{~Hz}$ was chosen to occur at an impedance minimum so that the effect on the next maximum can be more easily distinguished. Lattices with 10 holes exhibit very strong cutoff behavior. .................

3 Multiple simulated input impedances for resonators with acoustically regular lattices and random cell orders (50 permutations). The target frequencies are $f_{1}=170 \mathrm{~Hz}$ and $f_{\mathrm{c}}=1350 \mathrm{~Hz} \ldots \ldots \ldots \ldots \ldots \ldots \ldots \ldots \ldots \ldots \ldots \ldots \ldots \ldots \ldots$

4 Input impedance of $\mathcal{R}_{1.0}$ (a) and $\mathcal{R}_{2.0}$ (b). Grey: TMM simulation; dashed black: TMMI simulation; solid black: experimental. ................

5 Synthesized (dimensionless) pressure (top) and flow (bottom) inside mouthpiece for resonators $\mathcal{R}_{1.0}$ (solid black), $\mathcal{R}_{1.5}$ (dash-dot gray), and $\mathcal{R}_{2.0}$ (dashed black) in the time (left) and frequency (right) domains. Control parameters are $\gamma=\zeta=0.45$, and reed resonance $f_{r}=1500 \mathrm{~Hz} \ldots \ldots \ldots \ldots \ldots$

6 Synthesized playing frequency in cents, normalized by the first impedance peak frequency, for resonators $\mathcal{R}_{1.0}, \mathcal{R}_{1.5}$, and $\mathcal{R}_{2.0}$ with solid black, solid grey, dashed black lines, respectively........................

$7 \quad$ Synthesized spectral centroid, normalized by the playing frequency, for resonators $\mathcal{R}_{1.0}, \mathcal{R}_{1.5}$, and $\mathcal{R}_{2.0}$ with solid black, solid grey, dashed black lines, respectively. 


\section{Effect of cutoff frequency on woodwinds}

8 Synthesized $(\zeta=0.45, \gamma=0.55)$ spectra of the mouthpiece pressure for resonators $\mathcal{R}_{1.0}, \mathcal{R}_{1.5}$, and $\mathcal{R}_{2.0}$ for 20 fundamental frequencies ranging chromatically from $110 \mathrm{~Hz}$ to $330 \mathrm{~Hz}$. Odd and even harmonics denoted by squares

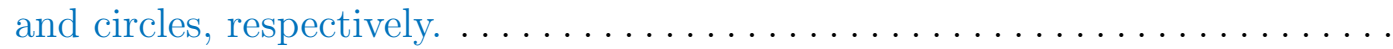

9 Time averaged intensity passing through the entrance of the resonator (left) and the summed over ten toneholes and exit of resonator (right). Resonators and synthesis control parameters are the same as in Fig 8. The cutoff frequency for each set of resonators is marked by a vertical line. . . . . . . . .

10 Synthesized pressure waveform and sum of time evolving harmonics for resonator $\mathcal{R}_{1.0}$ with $5 \%$ and $95 \%$ thesholds marked. . . . . . . . . . .

11 Synthesized attack time normalized by period of playing frequency for resonators $\mathcal{R}_{1.0}, \mathcal{R}_{1.5}$ and $\mathcal{R}_{2.0}$ with solid black, solid grey, dashed black lines, respectively. Variation in attack times due to cutoff frequency is generally

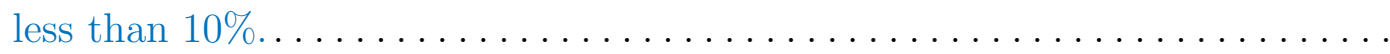

\section{List of Tables}

I Dimensions for two prototype resonators with the first impedance peak $f_{1}$ at $170 \mathrm{~Hz}$ and cutoff frequencies $f_{\mathrm{c}}$ at $1000 \mathrm{~Hz}$ and $2000 \mathrm{~Hz}$. Simulated and measured input impedance are provided in Fig. $4 . \ldots \ldots \ldots \ldots \ldots \ldots$

II Frequencies of the first two impedance peaks and inharmonicity of resonators

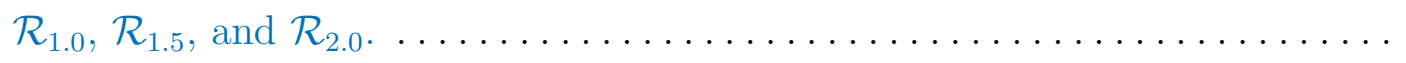

